www.ibpas.com

\title{
CHRONIC DISEASE PATIENT HEALTH GUIDANCE FRAMEWORK USING ARTIFICIAL INTELLIGENCE
}

\author{
RAJKUMAR N ${ }^{1}$, SAMUEL PAUL ISAAC ${ }^{2}$ AND ANURADHA N ${ }^{3}$
}

1: Department of Computer Applications, Krupanidhi Group of Institutions, Bengaluru, Karnataka

2: Department of Management, Krupanidhi Group of Institutions, Bengaluru, Karnataka

3: Department of Computer Applications, Krupanidhi Degree College, Bengaluru,

Karnataka

*Corresponding Author: Dr. Rajkumar N; E Mail: rajkumar@krupanidhi.edu.in

Received $19^{\text {th }}$ Aug. 2021; Revised 20 ${ }^{\text {th }}$ Sept. 2021; Accepted $29^{\text {th }}$ Oct. 2021; Available online $1^{\text {st }}$ Dec. 2021

https://doi.org/10.31032/IJBPAS/2021/10.12.2027

\begin{abstract}
Chronic disease management is expensive and time-consuming for world healthcare systems due to sufferers' strong desire for continual attention. As a result of these factors, patients are compelled to visit their doctors more regularly; it is impossible to keep track of many uncontrolled, ineffective visits. The present chronic disease is continuous efforts by both the patient and the health care providers to collect, investigate, and then alter management procedures. The usage of technology and Artificial Intelligence has eased these operations. Rarely has research sought to incorporate artificial intelligence-based designs into health education efforts for patients with chronic diseases. Numerous countries have promoted fitness guidance as a vital component of their public health policy. Health science is increasingly being used to encourage people to adopt better habits. This paper combined healthcare guidance with artificial intelligence to support patients to stick and manage their long-term care management.
\end{abstract}

Keywords: Health guidance, Artificial Intelligence, Chronic Disease

\section{INTRODUCTION}

One of the most confusing issues affecting global healthcare systems is a chronic disease. According to the 2011-2018 annual report [1], chronic illnesses might be acute 
or long-term. Unlike acute disorders, which have evident symptoms and a short duration, chronic diseases require much care because they last a long time, not just a short time [2]. The United States has spent $\$ 1.65$ trillion on chronic illness, accounting for $75 \%$ of the country's annual healthcare costs.

Moore studied the use of generation in treating chronic disorders [3] and realized that the most crucial task was to keep the illness from returning. Successful management software must not only be checked out regularly, but it must also be able to change with the patient's needs. As a result, because the indications of the control method change and emerge over time, they cannot be examined during a 30minute visit to the doctor's office. Variations occur throughout the patient's everyday routines rather than at the physician's clinical office. Measurements based on episodic patterns of episodic visits become inefficient as a result. Patient input, according to Moore, is the most overlooked aspect of illness control.

To begin, we will look at some essential terminology to better grasp the goal and model of this paper. The National Institutes of Health define fitness education as $\mathrm{a}$ supplemental medical intervention meant to induce healthy behavior modification and have an effect on the prevalence of chronic lifestyle disorders" [4]. Chronic diseases are those that last for an extended period. "There is no cure or specialist therapy for scientific illnesses. Care is geared toward sustaining a high standard of living for as long as possible" [5].

The goal of these works of art is twofold:

- Examine the current state of knowledge about the use of artificial intelligence (AI) in health guidance.

- Second, the goal is to improve health education that uses Persuasive Technology.

Advancements in e-health technology and sensors that enable measuring multiple aspects of human fitness have increased interest in this field of study [6]. Furthermore, most e-health gadgets use AI without fully comprehending the ramifications [7]. As a result, determining how AI may be integrated into sickness treatment strategies is crucial [8]. Paintings from this era illuminate experiments that incorporated artificial intelligence and health guidance to inspire and motivate people suffering from chronic diseases to stick to their treatment programs.

\section{LITERATURE REVIEW}

We discuss the importance of artificial intelligence in providing health advice to individuals living with chronic diseases. There is no clear description of health education in the evaluated articles or 
treating chronic diseases. However, as specified in the preceding stage, the study assessed the consistent criteria for health guidance. We begin by comparing and contrasting patients' identities and interactions with sensors, mobile devices, and software applications across the many chronic conditions we studied. We will first review a range of models and ideas that have been employed in the current framework of studies to create the groundwork for the model that we can expand.

\section{Crucial Treatment Aspects for Chronic}

\section{Diseases}

To gain a comprehensive understanding of the long-term sickness control system, we investigated eleven distinct programs that dealt with eleven different types of longterm illnesses, ranging from cardiovascular disorders to Parkinson's disease to multiple sclerosis [9] [10, 11]. For example, we looked at studies that looked at the same long-term conditions (e.g., diabetes) but used a range of different ideas to design their trials. This decoupling method allowed us to study the findings of each concept individually and provided insight into how we could merge the many ideas to construct a more coherent model.

MacLeod discovered persons suffering from unusual chronic illnesses and investigated how their ailments shaped their personalities $[12,13]$. MacLeod has conducted more studies on patients' perceptions toward their medical equipment. She employed semi-established interview chains and one-of-a-kind sensors to see if her experiment succeeded. Patients will not use technology if they do not believe that the sensors, mobile applications, or other technology understand their daily attempts to better their condition. Finding was obtained via a unique investigation exploring unusual and persistent illnesses.

Nunes has carried out experiments similar to MacLeod's on Diabetes and Parkinson's patients [12 - 14]. He researched a variety of additive technologies. AI-powered patient and clinician devices, as well as communication platforms, are examples of this. According to Nunes [14-16], generation should promote patient autonomy and collaboration. MacLeod's [13] is an example of humans and devices sharing information.

Theories Of Health Guidance Framework For Chronic Patients

Used many concepts in rare research, and their benefits were outstanding in exceptional testing using multiple AI modalities and chronic disorders. Moore's cognitive apprenticeship theory and Fogg's persuasive technology theory developed this model. Some studies expressly 
acknowledge these concepts (for example, diabetes [17]), whereas others use them without mentioning them for diabetes [18]. The use of technology to influence customer behavior is known as persuasive technology. There are various approaches to modeling the human-computer connection (whether a sensory form or an AI shape, for example) to inspire humans to perform extraordinary tasks (in our case, top health-associated conduct). Fogg [16] proposes the following criteria: The term "Functional Triad" refers to how humans perceive and respond to computing technology, whether as gear, media, or social actors, or all three at the same time.

According to him, Moore's notion of apprentice delivery [3] is a patient-centered approach to chronic illness care. The constant digital contact between the patient and the clinician will allow common data usage and analysis, increasing patient communication and understanding of longterm issues. Sufferers are essentially doctors' stooges. Patients who use technology to cure chronic diseases become "masters of their craft" due to regular interaction with technology and appropriate clinician training. Patients frequently understand their wishes better than the era or doctors, and clinician training is required to link their preferences with therapeutic demands.

\section{Outcomes and Insights}

We wanted to examine more about the impact of these concepts on chronic disease patients. As a result, we selected studies that used the same form of communication (mobile), a comparable range of device capabilities, a comparable time, and participants with the same chronic condition (Diabetes). Additionally, we used Orji [18]'s study of the persuasive writing influence on self-care packages to get insight into other prevalent circumstances and ideas, such as the two described in the preceding section of related works. On the other hand, Orji is no longer limited to debating persuasive technologies.

In an indirect application of the cognitive apprenticeship and persuasive generation theories, Hsu [17] employed a software platform for distributing programs to mobile phones and tablets as a scaffolding for cooperation in a study. As a consequence of this platform's tracking tools, visualizations, and personalized decision-making support, users better know how their behaviors and outcomes are linked. While the program primarily used $\mathrm{AI}$ and ML to collect and process data, the system enhanced the apprenticeship concept by allowing patients to obtain private education from physicians via faceto-face or digital communication. Patients rapidly accepted and embraced machine 
learning recommendations once they understood the application's physics. According to two independent reviews [19] and [20], the vast majority of current AI systems are either unaware of or unable to identify the amount of cognitive processing and machine learning control that patients require. Consumers will forsake the item even if it performs well without user control. As a result of their research, they came to this conclusion. As a result, a guide or mentor is needed to bridge the patient and the technology. The instructor could be either a person or a machine function.

Consider [21] based on Fogg's clear technology idea. DenAkker created a device for diabetic teens that uses gamification to help them regulate their diabetes regularly. The most important lesson from the study's termination results was users' persistence in using the software after the examination period expired. DenAkker ensured the software's clients' compliance by requiring the device to perform three primary functions [22]. The first goal is to connect the system to a glycemic study tool to measure blood glucose levels automatically and often. The second goal was to help people with diabetes manage their condition by employing educational games, monitoring, and positive reinforcement [23-27]. Third, a created virtual guidance in an interactive gaming guide to delivering individualized patient feedback. Each of these three jobs fulfilled one of Fogg's [16] three components of the purposeful triangle. Using Orji's [18] systematic examination, we extensively investigated several applications for various chronic conditions.

Chronic Disease Patient Health Guidance Framework: An Artificial Intelligence Application Model

This section goes over the model that employs artificial intelligence and other cutting-edge technology to assist patients suffering from recurring diseases build a personalized treatment plan, as shown in

\section{Figure 1.}

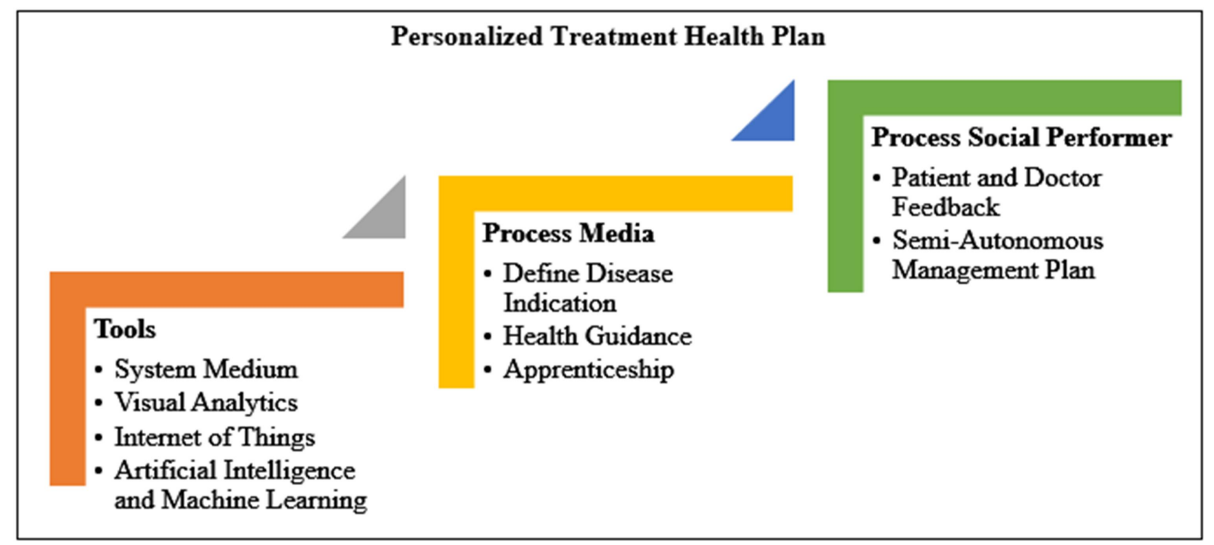

Figure 1: Chronic Disease Patient Health Guidance Framework: An Artificial Intelligence Application Model 
Health guidance can help patients with chronic diseases using AI. Patients suffering from a specific chronic disease can benefit from adopting this model to develop a personalized treatment plan by adhering to the variety's markers. There are different steps of building blocks in this figure.

A tactic is defined as a series of acts designed to attain a specific objective. The pinnacle phases are crucial for establishing a personalized training program due to these adventure shaping strategies. Each component is stacked on top of the others. Several strategies are as follows: The media process and the social performers' tactics. The three-step methods correlate to the three components of Fogg's functional triangle. By incorporating all three components of the beneficial triangle into our model, we could replicate the conclusion following the outcome and the insights gained from the literature research.

\section{Tools}

The step comprises four building elements, each essential to the system's functionality. Each piece of equipment is briefly discussed below.

System Medium: First, the system medium serves as the period system's operational platform. Choose it carefully if you want to get the most out of this platform. According to West [19], system medium is defined by the nature of data and how it must be displayed, rather than the other way around. The medium constrains how the data is visualized. This is extremely important to remember. As a result, the machine medium device at the top of the gear step is positioned.

Visual Analytics: Visual analytics solutions are critical for efficiently communicating statistical data in the appropriate quantity and manner to patients and physicians. These technologies provide the most relevant information to both the patient and the physician. When it comes to deciding whether to deliver data in a visual or written style, physicians can rely on these tools to help them make the most effective use of their patients' limited cognitive resources. This item is connected to the next with considerable caution.

Internet of Things: The Internet of Things forms the basis of the equipment hierarchy. They are the first component built-in every model since they generate its data. When a patient is identified with a chronic disease, the symptoms and sensors that go with it are uncovered.

Artificial Intelligence and Machine Learning: $\mathrm{AI}$ and $\mathrm{ML}$ relieve doctors and patients of a load of diagnosing and forecasting symptoms of chronic diseases by immediately impacting the demanding settings that chronic disease patients 
confront in the early stages (i.e., Endless repetitive quick visits, the lack of time for clinicians, and the ineffectiveness of these visits). Consultation with a physician will be more beneficial now that machine learning and artificial intelligence gadgets may assist physicians with insights and their selection decisions.

\section{Processes Media}

It is made up of two steps, each of which represents a different strategy critical to the success of the steps.

In addition to media processes, these methods, in particular, are intended to provide customers with the knowledge they need to understand the influence of their actions on the world around them [16].

Defining the Disease Indications: The clinician is the most critical person determining disease signs. Based on the nature of the patient's long-term sickness, the doctor chooses a set of symptoms that sensors can monitor. When correctly explored, AI and machine learning can provide valuable insights about the success of changing and customizing the care plan for a patient with a chronic condition in the country in which the patient lives. As a result, it is crucial to remember that symptoms might vary greatly depending on the underlying condition. The term "indications" refers to both vital signs and symptoms and those unique to a specific illness and are physiological or psychological.

Health Guidance: As part of the health guidance strategy, the educated clinician or nurse conducts a series of brief education sessions and lectures to assist the patient in self-educating about the recurrent issue. After completing the health education program, the patient should recognize symptoms of illness and make required adjustments to their condition. This approach is related to the one that came before it. It initiates an apprenticeship in chronic disease treatment, with the physician serving as the "master of the art" and the patient as the "disciple."

Apprenticeship: This technique builds on the previous one, in which the patient learned the principles of chronic disease management. Nonetheless, the patient had exhausted all available generations to administer the common issue properly. Patients are allowed to use a gadget multiple times with the assistance of a physician until they understand its capabilities and can connect their medical history learned in health education classes to those functions through generation apprenticeship.

\section{Processes Of Social Performers}

These approaches are designed primarily to improve communication and collaboration between the patient, the physician, and any 
other parties involved in their treatment.

Patient and Doctor Feedback: To achieve this stage, the patient must have "mastered" both the fitness and technological aspects of his chronic disease. As a result, a feedback involving both the physician and the machine is now possible. The patient's positive observations and personalization's will be sent to the therapist and device, ensuring that the health plan is customized to the patient's particular preferences.

Semi-Autonomous Management Plan: The patient is semi-autonomous in treating their ongoing sickness at this time, with only a few visits to their doctor. As a result, the patient has more time to think about and analyze the information provided by the technological device, allowing him to understand better the nature of his chronic condition and how to treat it successfully. As a result of the customizing process, the patient now has a more thorough grasp of their ailment than they had previously. The data will help patients better understand their situation, allowing them to manage it more efficiently and autonomously. Although this concept has not yet been implemented, it is expected to improve medication adherence, hospitalizations, and emergency room visits.

\section{CONCLUSION}

AI-supported Health Guidance described a health guidance technique for chronic diseases that blends cognitive apprenticeship theory and persuasive technology. We believe that our approach is the first of its type to combine all three practical triad components of Fogg's persuasive generation notion and $\mathrm{AI}$ as a tool for giving physical training and cognitive apprenticeships, based on the literature. We have outlined three different ways that can use the offered artwork. This is done by providing complete instructions for each piece of equipment and all recommended adjustments. Suggestions can help with method support, enhancing stability by restricting the number of approaches and tools available. Second, the proposed HCI model can benefit from a system that learns about subversion through experimentation with scientific records for a specific chronic disease by creating a test and a mechanism for determining the efficacy of the proposed model. Once the observer results are gathered, the model's strengths and limitations and chances for improvement.

\section{ACKNOWLEDGMENT}

The authors express gratitude towards the assistance provided by The Management, Krupanidhi Group of Institutions (KGI) and Krupanidhi Research Incubation Centre, KGI in completing the research. We also thank our Research Mentors who guided us throughout the research and helped us in 
achieving the desired results.

\section{REFERENCES}

[1] Amershi S, Weld D, Vorvoreanu M, Fourney A, Nushi B, Collisson P, Suh J, Iqbal S, Bennett PN, Inkpen K, Teevan J. Guidelines for human-AI interaction. InProceedings of the 2019 chi conference on human factors in computing systems 2019 May 2 (pp. 113).

[2] Angehrn Z, Haldna L, Zandvliet AS, Gil Berglund E, Zeeuw J, Amzal B, Cheung SY, Polasek TM, Pfister M, Kerbusch T, Heckman NM. Artificial intelligence and machine learning applied at the point of care. Frontiers in Pharmacology. 2020 Jun 18;11:759.

[3] Battineni G, Sagaro GG, Chinatalapudi N, Amenta F. Applications of machine learning predictive models in the chronic disease diagnosis. Journal of personalized medicine. 2020 Jun;10(2):21.

[4] Denecke K, Gabarron E, Grainger R, Konstantinidis ST, Lau A, RiveraRomero O, Miron-Shatz T, Merolli M. Artificial intelligence for participatory health: applications, impact, and future implications. Yearbook of medical informatics. 2019 Aug;28(01):165-73.

[5] Dermody G, Fritz R. A conceptual framework for clinicians working with artificial intelligence and health- assistive Smart Homes. Nursing inquiry. $2019 \mathrm{Jan} ; 26(1): \mathrm{e} 12267$.

[6] Fan D, Ren A, Zhao N, Yang X, Zhang Z, Shah SA, Hu F, Abbasi QH. Breathing rhythm analysis in body centric networks. IEEE Access. 2018 Jun 12;6:32507-13.

[7] Fogg BJ. Persuasive technology: using computers to change what we think and do. Ubiquity. $2002 \quad$ Dec 1;2002(December):2.

[8] Haider D, Ren A, Fan D, Zhao N, Yang X, Tanoli SA, Zhang Z, Hu F, Shah SA, Abbasi QH. Utilizing a 5G spectrum for health care to detect the tremors and breathing activity for multiple sclerosis. Transactions on Emerging Telecommunications Technologies. 2018 Oct;29(10):e3454.

[9] Hsu WC, Lau KH, Huang R, Ghiloni S, Le H, Gilroy S, Abrahamson M, Moore J. Utilization of a cloud-based diabetes management program for insulin initiation and titration enables collaborative decision making between healthcare providers and patients. Diabetes technology \& therapeutics. 2016 Feb 1;18(2):59-67.

[10] Khan MB, Yang X, Ren A, Al-Hababi MA, Zhao N, Guan L, Fan D, Shah SA. Design of software defined radios based platform for activity recognition. IEEE Access. 2019 Feb 28;7:31083-8. 
[11]MacLeod H, Bastin G, Liu LS, Siek K, Connelly K. " Be Grateful You Don't Have a Real Disease" Understanding Rare Disease Relationships. InProceedings of the $2017 \mathrm{CHI}$ Conference on Human Factors in Computing Systems 2017 May 2 (pp. 1660-1673).

[12] MacLeod $H$, Oakes $K$, Geisler $D$, Connelly K, Siek K. Rare world: Towards technology for rare diseases. InProceedings of the 33rd Annual ACM Conference on human factors in computing systems 2015 Apr 18 (pp. 1145-1154).

[13]Mohanta B, Das P, Patnaik S. Healthcare 5.0: A paradigm shift in digital healthcare system using artificial intelligence, IOT and 5G communication. In2019 International Conference on Applied Machine Learning (ICAML) 2019 May 25 (pp. 191-196). IEEE.

[14]Moore JO. Technology-supported apprenticeship in the management of chronic disease (Doctoral dissertation, Massachusetts Institute of Technology). 2013.

[15]Morley J, Floridi L. An ethically mindful approach to AI for health care. Available at SSRN 3830536. 2020.

[16]Nunes F, Fitzpatrick G. Understanding the mundane nature of self-care:
Ethnographic accounts of people living with Parkinson's. Inproceedings of the 2018 CHI Conference on Human Factors in Computing Systems 2018 Apr 21 (pp. 1-15).

[17] Nunes F, Verdezoto N, Fitzpatrick G, Kyng M, Grönvall E, Storni C. Selfcare technologies in HCI: Trends, tensions, and opportunities. ACM Transactions on Computer-Human Interaction (TOCHI). 2015 Dec $14 ; 22(6): 1-45$.

[18] Nunes F. Designing self-care technologies for everyday life: A practice approach. InProceedings of the 33rd Annual ACM Conference Extended Abstracts on Human Factors in Computing Systems 2015 Apr 18 (pp. 215-218).

[19] Oikonomou EK, Siddique M, Antoniades C. Artificial intelligence in medical imaging: a radiomic guide to precision phenotyping of cardiovascular disease. Cardiovascular research. 2020 Nov 1;116(13):204054.

[20] Orji R, Moffatt K. Persuasive technology for health and wellness: State-of-the-art and emerging trends. Health informatics journal. 2018 Mar;24(1):66-91.

[21] Rana JS, Khan SS, Lloyd-Jones DM, Sidney S. Changes in Mortality in Top 
10 Causes of Death from 2011 to 2018.

Journal of general internal medicine. 2020 Jul 23:1-2.

[22] Schachner T, Keller R, Von Wangenheim F. Artificial intelligencebased conversational agents for chronic conditions: systematic literature review. Journal of medical Internet research. 2020;22(9):e20701.

[23] Sforzo GA. The study of health coaching: the Ithaca coaching project, research design, and future directions. Global advances in health and medicine. 2013 May;2(3):58-64.

[24] Varin M, Baker M, Palladino E, Lary T. At-a-glance-Canadian Chronic Disease Indicators, 2019-Updating the data and taking into account mental health. Health promotion and chronic disease prevention in Canada: research, policy and practice. 2019 Oct;39(10):281.

[25] West VL, Borland D, Hammond WE. Innovative information visualization of electronic health record data: a systematic review. Journal of the American Medical Informatics Association. 2015 Mar 1;22(2):330-9.

[26] Yang X, Fan D, Ren A, Zhao N, Shah SA, Alomainy A, Ur-Rehman M, Abbasi QH. Diagnosis of the Hypopnea syndrome in the early stage. Neural Computing and Applications.
2020 Feb;32(3):855-66.

[27] Yang X, Shah SA, Ren A, Fan D, Zhao N, Cao D, Hu F, Rehman MU, Wang W, Von Deneen KM, Tian J. Detection of essential tremor at the $\$ \mathrm{~s} \$$-band. IEEE journal of translational engineering in health and medicine. 2018 Jan 24;6:1-7. 